\title{
HELIAS Module Development for Systems Codes
}

\author{
F. Warmer ${ }^{\mathrm{a}, *}$, C.D. Beidler ${ }^{\mathrm{a}}$, A. Dinklage ${ }^{\mathrm{a}}$, K. Egorov ${ }^{\mathrm{a}}$, Y. Feng ${ }^{\mathrm{a}}$, J. Geiger ${ }^{\mathrm{a}}$, F. Schauer ${ }^{\mathrm{a}}$, Y. Turkin ${ }^{\mathrm{a}}$, R. Wolf ${ }^{\mathrm{a}}$, \\ P. Xanthopoulos ${ }^{\text {a }}$ \\ ${ }^{a}$ Max Planck Institute for Plasma Physics, D-17491, Greifswald, Germany
}

\begin{abstract}
In order to study and design next-step fusion devices such as DEMO, comprehensive systems codes are commonly employed. In this work HELIAS-specific models are proposed which are designed to be compatible with systems codes. The subsequently developed models include: a geometry model based on Fourier coefficients which can represent the complex 3-D plasma shape, a basic island divertor model which assumes diffusive cross-field transport and high radiation at the X-point, and a coil model which combines scaling aspects based on the Helias 5-B reactor design in combination with analytic inductance and field calculations. In addition, stellarator-specific plasma transport is discussed. A strategy is proposed which employs a predictive confinement time scaling derived from 1-D neoclassical and 3-D turbulence simulations.

This paper reports on the progress of the development of the stellarator-specific models while an implementation and verification study within an existing systems code will be presented in a separate work.

This approach is investigated to ultimately allow one to conduct stellarator system studies, develop design points of HELIAS burning plasma devices, and to facilitate a direct comparison between tokamak and stellarator DEMO and power plant designs.
\end{abstract}

Keywords: HELIAS, stellarator-specific models, Systems Code

\section{Introduction}

With ITER [1] under construction, design studies now concentrate on a fusion facility which is to follow ITER. This follow-up step is often referred to as 'DEMO', short

5 for demonstration fusion power plant with the aim of demonstrating the technical maturity of the magnetic confinement fusion concept. But even the conceptual design of such a power plant like fusion device is a complex and demanding task. Therefore, to facilitate such studies, socalled 'systems codes' are often employed as valuable tools for the design process.

Systems codes, also known as design codes, are comprehensive yet simplified models of a complete fusion facility. Since they bring together physics, engineering and economic aspects as outlined above, self-consistent design points can be developed and their sensitivity against variation of critical parameters tested. With this approach especially critical development directions for physics scenarios or technology advancements can be identified. Following this, dedicated experiments and simulations may be performed and as a result of that, systems codes models updated, as is conceptually shown in Fig. 1 below.

For the tokamak concept, design activities have progressed lately employing different systems codes world- broad range of devices and corresponding possibilities have been studied, but also in the European PPCS studies [3, employing the systems code PROCESS 44, different tokamak scenarios have been investigated. Also basic power balance models can be employed to assess the required size of DEMO, taking also into account pulsed devices [5]. Many additional tokamak systems codes and studies exist, but it is beyond the scope of this work to cover all of them.

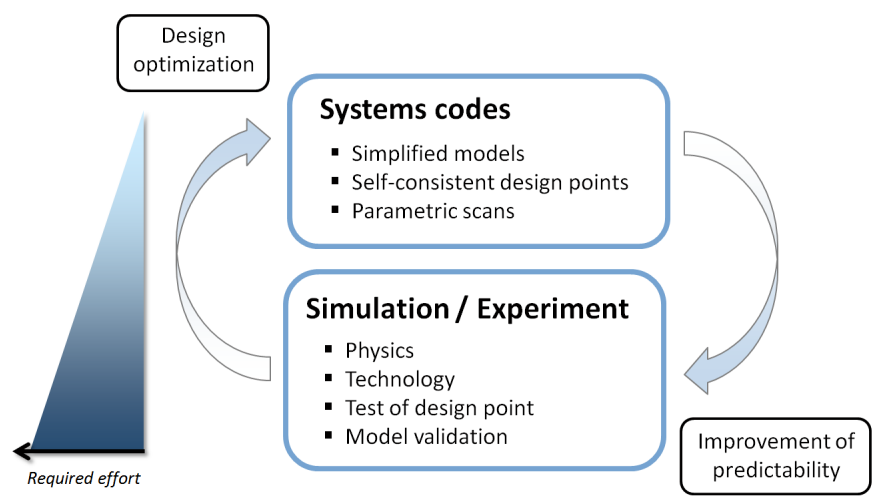

Figure 1: Concept of systems codes and their interaction with detailed simulations and experiments. The left scala illustrates the required effort (in terms of complexity and time) to carry out the individual tasks.

Systems codes have also been employed outside the toka35 mak community for the conceptual design of a heliotron

*Corresponding author, Tel.: +49 (0)3834 88-2583

Email address: Felix.Warmer@ipp.mpg.de (F. Warmer) 
DEMO. In contrast to the tokamak, the heliotron magnetic 90 field is created by continuous helical coils. This means the $3 \mathrm{D}$ effects of the helical coils and plasma shape must be considered, introducing additional complexity compared 40 to axissymmetric tokamaks. This is done in the design approach within the heliotron systems code HELIOSCOPE 95 [6] which led to the concept of a Force Free Helical Reactor DEMO (FFHR-d1) 7, 8].

Another magnetic confinement concept is the helical advanced stellarator (HELIAS). The HELIAS is a modular stellarator concept with periodic symmetry including inte-100 grated optimisation of the magnetic field with respect to several criteria at the same time, e.g. Shafranov shift, neoclassical transport, etc., where the magnetic field is established by extneral non-planar coils. But so far no systems code exists capable of modelling a HELIAS. Therefore this 105 work concentrates on the development of a HELIAS systems code module with the aim of implementation in the systems code PROCESS. PROCESS is a well-established,

${ }_{55}$ partly modular, tokamak systems code which gained maturity through many applications. A solver based on La-110 grangian multipliers is employed within PROCESS to allow for design optimisation with respect to the descriptive models and constraints. Such an approach is followed to ${ }_{60}$ allow for stellarator systems studies and design point development of HELIAS burning plasma devices as well as115 comparative studies to tokamaks.

The purpose of this work is to report on the progress of the development of HELIAS models for systems codes and

65 is organised as follows: In section 2 the essential differences between tokamak and stellarator are identified which re-120 quire preparation of new systems code modules. The corresponding models are described in section 3 which include a geometry model based on Fourier coefficients, a basic

70 island divertor model which assumes cross-field transport and high radiation, and a model for the non-planar, modu-125 lar coils based on scaling aspects with respect to the Helias 5-B reactor design [9] in combination with analytic calculations. Furthermore, stellarator-specific plasma transport

75 is discussed and a strategy proposed for the development of a predictive confinement time scaling. The work is sum-130 marised and the results are discussed in section 4. A detailed verification study of the HELIAS module will be presented in a seperate work [10] where the models have so been implemented in the systems code PROCESS.

\section{Identification of Required Models}

The tokamak and the stellarator differ in the point of how the rotational transform is created. In the tokamak $k_{140}$ this is done by driving a toroidal current in the plasma. The helical advanced stellarator concept, considered here, in contrast twists the magnetic field by poloidally rotating the elongated flux surfaces around a non-planar magnetic axis achieved exclusively by a set of non-planar modular ${ }_{145}$ coils. This fundamental difference has several implications:
A tokamak may only be operated as long as a current is driven in the plasma, which is either limited in time by the available magnetic flux, if driven inductively, or requires a large amount of power if driven non-inductively, e.g. by neutral beam injection (NBI). The HELIAS, in contrast, operates intrinsically steady-state, 'current-free' and without disruptions. 'Current-free' means that HELIAS configurations are optimised with respect to minimal bootstrap and Pfirsch-Schlüter currents and the net toroidal current is, therefore, several orders of magnitude below tokamak levels.

As the poloidal field component in the tokamak is created by the plasma current, the toroidal field component is achieved by planar, identical, typically 'D-shaped' coils. This makes the tokamak plasma shape (flux-surfaces) axisymmetric. The plasma geometry of the stellarator, in contrast, is fully three-dimensional with a periodic symmetry. Also the stellarator coils for the HELIAS line are 3D, non-planar, modular, and comparably numerous.

The complex 3D shaping of the stellarator magnetic field structure generally introduces localised helically trapped particle orbits which have an overall impact on the plasma transport. The resulting, so-called, 'neoclassical' transport can be very high in stellarators and is an essential optimisation criterion of helical advanced stellarators. Usually also an ambipolar electric field arises connected to the neoclassical transport. Additional 3D anomalous transport must be considered making the description of stellarator transport a complex task, especially since 3D turbulence simulations for stellarators have just been started. In tokamaks, in contrast, the turbulent transport is observed to be dominant drawing on a solid base of experiments and experience. In addition, tokamaks are geometrically similar which more confidently allows to describe the transport by empirical confinement time scalings based on the similarity principle.

Last, but not least, the axisymmetry of the tokamak allows to employ a toroidally closed divertor, either only on the bottom (so-called 'single-null') or up-down symmetric (so-called 'double-null'). In the HELIAS concept a chain of naturally occuring magnetic islands at the plasma edge is employed. Independent divertor plates are placed symmetrically at the top and bottom of each module intersecting the magnetic islands at the edge in order to efficiently control the particle and energy exhaust. From a tokamak viewpoint this could be seen as a discontinuous multi-null divertor.

In order to identify specific and independent models which need to be developed for a HELIAS systems code approach, the general considerations from above need to be checked with an existing systems code. For this purpose the well-developed and commonly employed systems code PROCESS has been selected. The source code of PROCESS and its corresponding tokamak models have been thoroughly reviewed with respect to stellarator-specific considerations. From this investigation it is concluded that in a systems code such as PROCESS, the plasma geome- 
try, the modular coils, the island-divertor and the plasma transport models require independent treatment compared to the tokamak models while treatment of current-related aspects can be neglected. The correspondingly developed ${ }^{195}$ models are described in the next section.

\section{The Stellarator Module}

In the following stellarator-specific models are proposed which are designed to be applicable to systems codes and which together build up a consistent HELIAS module. One requirement of this development is to retain small calculation times without compromising the necessary ac-205 curacy and complexity of the 3D stellarator-specific properties.

\subsection{Plasma Geometry}

At finite normalised plasma pressure $\langle\beta\rangle=2 \mu_{0}\left\langle p / B^{2}\right\rangle^{210}$ the shape of the confined plasma is determined by the shape of the nested closed flux surfaces (in stellarators even at $\langle\beta\rangle=0)$. In position-space those surfaces may be represented by cylindrical coordinates $(R, \varphi, z)$, but in practice it is more convenient to decompose these coor- ${ }^{21}$ dinates in a Fourier series with respect to poloidal and toroidal angle coordinates, respectively $u$ and $v$ as wells as the flux surface label $s$ :

$$
\begin{aligned}
R(s, u, v) & =\sum_{m=0}^{m_{\max }} \sum_{n=-n_{\max }}^{n_{\max }} R_{m, n}(s) \cos (m u-N n v)_{220} \\
z(s, u, v) & =\sum_{m=0}^{m_{\max }} \sum_{n=-n_{\max }}^{n_{\max }} z_{m, n}(s) \sin (m u-N n v)
\end{aligned}
$$

where $N$ is the number of field periods and $\varphi(s, u, v)$ is defined in the same way as $R$ and $z$.

Using this representation allows one to accurately cal- ${ }^{225}$ culate the important geometrical parameters relevant for the systems analysis by summation over the correspondcombination of Fourier coefficients. The important geometrical parameters are the effective average plasma cross-sectional area $\langle F\rangle$ in $\mathrm{m}^{2}$, the plasma volume $V$ in $^{230}$ $\mathrm{m}^{3}$ as well as the total surface area of the confined plasma $S$ in $\mathrm{m}^{2}$. These parameters are important due to their direct impact on relevant physics and engineering quantities, e.g. the volume for the confinement time and fusion power or the surface area for neutron wall load.

In order to employ and use this model, the Fourier coefficients $R_{m, n}$ and $z_{m, n}$ of the last closed flux surface (LCFS) be provided. These can be obtained from equilibrium calculations employing e.g. the equilibrium code VMEC.

The advantage of this model is its generality as every arbitrary toroidal shape can be treated, including tokamak ${ }^{240}$ and heliotron geometry. Moreover, it is possible to scale both the minor and major plasma radius by scaling of the corresponding Fourier coefficients making it very flexible while sustaining the general shape.

\subsection{Island Divertor}

For tokamaks, often so-called 2-point models are used to describe the particle and energy transport from an upstream position parallel along the magnetic field to the divertor target. For stellarators, in contrast, such 2-point models are more complex and additional terms and parameters must be considered [11, 12]. However, the correlations of some of these parameters are to-date unclear. For this reason the 2-point model approach is avoided here. Instead, a more basic and fundamental model is proposed based on geometrical considerations. The model combines relations of physics as well as engineering and is therefore well suited for scaling. Another advantage of such a basic model is that parts of it can be easily replaced once a more detailed understanding, e.g. of the power decay width, is available.

The heat load on the divertor plates, $q_{\text {div }}$, is defined as the ratio of the power transported to the divertor, $P_{\text {div }}$, over an effective wetted area, $A_{\text {eff }}$. The total power arriving at the divertor is the power crossing the separatrix, $P_{\mathrm{SOL}}$, provided from the plasma transport model, less the SOL and X-point radiation: $P_{\mathrm{div}}=P_{\mathrm{SOL}}\left(1-f_{\mathrm{rad}}\right)$, where the radition fraction is contained in the factor $f_{\mathrm{rad}}$.

The wetted area can be seen as the product of the total length of all divertor plates, $L_{\mathrm{T}}$, and the power decay width, $\lambda_{q}$, at the divertor plate. Accounting additionally for some asymmetry with a factor $f_{a}$ the heat load becomes

$$
q_{\mathrm{div}}=\frac{P_{\mathrm{div}}}{A_{\mathrm{eff}}}=\frac{P_{\mathrm{SOL}}\left(1-f_{\mathrm{rad}}\right)}{L_{\mathrm{T}} \cdot \lambda_{q}} \cdot f_{a} .
$$

The total length $L_{\mathrm{T}}$ of the discontinuous island divertor is the sum over all identical divertor plates of which there are two in every field period, therefore $L_{\mathrm{T}}=2 n L_{\mathrm{D}}$ with the toroidal and poloidal mode number $n$ and $m$ respectively.

Here, the length of a single divertor plate $L_{\mathrm{D}}$ may be estimated from a geometric approach. Starting from the $\mathrm{X}$-point and following a flux tube, the field lines experience a radial pitch of angle $\Theta$ in the island region while going helically around the torus until striking the divertor plate as illustrated in Fig. 2, A field line which just passes the divertor plate on the inner side will strike the divertor plate at the far outer point after $m$ field periods. The radial distance from the inner to the outer side of the plate is then the helical length $2 \pi R m / n$ times the pitch angle $\Theta$. The radial extent is enhanced by diffusive broadening of the flux channel $F_{x}$ and the length of the divertor plate is then determined from the inclination of the divertor plate relative to the field lines $\alpha_{\text {lim }}$. Combining this, the wetted length of one divertor plate is

$$
L_{\mathrm{D}}=2 \pi R \cdot \frac{m}{n} \frac{\Theta}{\alpha_{\lim }} F_{x} .
$$

Both $F_{x}$ and $\lambda_{q}$ are attributed to cross-field transport. Two main assumptions are made in the island divertor model: 
- diffusive cross-field transport is considered (due to much longer connection lengths in stellarators compared to tokamks)

- high radiation fraction is assumed in the SOL and at the X-point (which is necessary to protect the divertor ${ }^{280}$ from severe heat loads)

From general diffusive transport behavior the power decay width is described by $\lambda_{q}=\sqrt{\chi_{\perp} \cdot \tau_{\|}}$with the perpendicular diffusion coefficient $\chi_{\perp}$ and the characteristic diffusion time $\tau_{\|}$which is determined by parallel transport.285 The second assumption made above implies a cold island in which the temperatures are so low that the remnant heat is mainly transported by convective processes onto the targets.

From the geometric view introduced above it is clear ${ }^{290}$ that the characteristic time for a flux channel to undergo diffusion is determined by the connection length from the $\mathrm{X}$-point to the target plate $L_{X \rightarrow T}$ and the ion sound speed $c_{s}=\sqrt{2 T / m}$ of the particles $\tau_{\|}=L_{X \rightarrow T} / c_{s}$. The connection length in turn may be estimated from the distance between X-point and divertor plate $\Delta$, as illustrated $\operatorname{in}_{295}$ pitch $\Theta$ by $L_{X \rightarrow T}=\Delta / \Theta$. The typical dimension of $\Delta$ is related to the radial width of the magnetic islands $w_{r}$ such that

$$
\Delta=f_{w} \cdot w_{r}=f_{w} \cdot 4 \sqrt{\frac{R \cdot b_{m, n}}{n \cdot t^{\prime}}}
$$

where $f_{w}$ is a fractional factor of order $1 / 2, b_{m, n} \sim \Theta$ the radial field perturbation, and $t^{\prime}$ the magnetic shear in the SOL. The flux channel broadening which determines the divertor plate length can be derived with the same general diffusive transport behavior with the connection ${ }^{305}$

$$
F_{x}=1+\frac{1}{\Theta} \sqrt{\frac{\chi \perp}{c_{s} 2 \pi R \frac{m}{n}}} .
$$

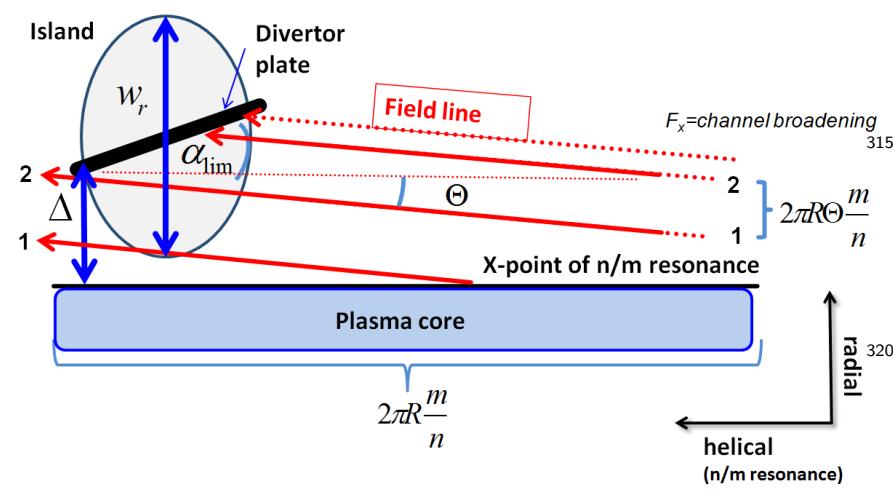

Figure 2: Geometrical illustration of a flux tube in the scrape-off 325 layer intersected by a divertor plate.

Due to the analytic nature of the model, the single aspects can be consolidated in a single formula for the head load with respect to the required input parameters as:

$$
q_{\mathrm{div}}=\frac{P_{\mathrm{SOL}}\left(1-f_{\mathrm{rad}}\right)}{4 \pi R m F_{X}} \frac{\alpha_{\text {lim }}}{\Theta \cdot \chi_{\perp}} \frac{1}{2} \sqrt{\frac{c_{s}}{f_{w}}} \sqrt[4]{\frac{n \cdot t^{\prime}}{R \cdot b_{m, n}}} \cdot f_{a}
$$

The input parameters are obtained from the envisaged reactor design, e.g. $R, P_{\mathrm{SOL}}$, the considered magnetic configuration, e.g. $\Theta, m, n$, as well as from experience from existing devices for engineering, e.g. $\alpha_{l i m}$, and physics, e.g. $\chi_{\perp}, c_{s}$.

It should be noted, that due to the basic nature of the model, the quantitative accuracy is limited and sensitive to the input parameters. The model should rather be interpreted to predict the dimension of the heat load. More importantly, as the heat load is usually limited by material constraints the model is useful to estimate the required radiation fraction $f_{\text {rad }}$ in order to ensure safe divertor operation for a specific design.

\subsection{Modular Coils}

Since the design of coils for an optimised stellarator configuration is a demanding process requiring complex codes, computational power and, experience, several major approximations must be considered to represent modular coils in a systems code approach:

- the sophisticated Helias 5-B reactor design study and its coil design are used as reference basis [9]

- the coil shapes of this design are assumed to be 'fixed' but the overall size shall be scalable

- based on physics principles, scaling factors and relations are introduced to flexibly scale the design according to a set of desired parameters

With the Helias 5-B coil design as basis and under the assumption of fixed coil shapes several scaling factors can be introduced with relation to Helias 5-B parameters. for the coil radius $f_{s}$. As HELIAS devices have closely positioned coils, the total coil current can be consequently scaled with $f_{I}=f_{B} \cdot f_{R}$ where $f_{B}$ is the scaling factor for the magnetic field strength on axis.

A semi-analytic method is employed, in order to calculate the maximal magnetic field on the surface of the coil, $B_{\max }$, the total stored magnetic energy, $W_{\text {mag }}$, as well as the cross-sectional dimension of the winding pack (WP). Each coil consists of $N$ turns placed in the winding pack as in Helias 5-B where the size of the WP is considered an input in the analytic description for the moment (but is later calculated self-consistently with an additional constraint). The turns are approximated by circular filaments. The mutual inductance, $M$, between two arbitrarily spaced and oriented circular filaments can then be calculated analytically 13 . 
The total inductance $L_{\text {tot }}$ is obtained by summation over 380 all mutual inductances of circular filaments and the selfinductances of the coils. The latter are simply approximated by the inducantance of a circular loop with circular cross section of radius $\left(A_{\text {sect }} / \pi\right)^{1 / 2}$, where $A_{\text {sect }}$ is the recangular winding pack section area. From this follows the385 total stored magnetic energy simply by $W_{\text {mag }}=\frac{1}{2} L_{\text {tot }} I^{2}$ with the total coil current $I$.

The magnetic field at any point in space can be straightforwardly calculated as sum of solenoid fields, using standard loop formulas and elliptic integrals as, e.g., found in 390 [14. This way the average magnetic field on the plasma axis as well as the maximal field, $B_{\max }$, at the coils can be found.

Until this point the dimensions of the winding pack were a free parameter, but the aim of this model is to selfconsistently calculate important parameters. For this purpose the critical current density behavior of the respective erconducting material may be employed as a natural constraint. Treating the coil winding packs as single current carrying conductors, the $\mathrm{Nb}_{3} \mathrm{Sn}$ ITER scaling [15] assuming constant operation temperature - can be simplified to

$$
f_{q}=10.9 \cdot \sqrt{f_{I}} \frac{B_{\max }^{1 / 4}}{33-B_{\max }}
$$

where $f_{q}$ is the scaling factor for the winding pack cross section area. The constant factor contains the critical field of the superconductor and the maximal field of Helias 5-B. With this constraint the magnetic energy and field calcuations given above can be iterated with respect to $f_{q}$ and determined self-consistently. The cross-sectional area of the winding pack is subsequently scaled by $f_{q}^{2}$ from which the radial and toroidal width of the WP can be obtained. But it should be noted that the WP aspect ratio of radial o toroidal extension is kept fixed according to Helias 5-B. Similar formulas can be derived for other superconductors such as e.g. NbTi.

Based on the magnetic field and the corresponding stored magnetic energy, the total required mass of support structure can be estimated. Since the Virial theorem links the magnetic energy and the minimal mass of support structure, this principle can be used to derive an empirical400 scaling between these parameters on the basis of existing superconducting devices, as e.g. demonstrated in [16]. By considering in addition the most recent superconducting devices such as W7-X, LHD, and ITER the empirical scaling of [16] is updated to

$$
M_{\mathrm{struc}}=1.3483 \cdot W_{\mathrm{mag}}^{0.7821}
$$

with the stored magnetic energy $W_{\text {mag }}$ in MJ and the mass of support structure $M_{\text {struc }}$ in t, illustrated in Fig. 3. It can be seen from the figure that several experiments, both tokamaks and stellarators, are well aligned with the em-410 pirical fit (blue line) reaching over several orders of magnitude. It should still be noted that the figure represents a double logarithmic plot and a single device may deviate up to a factor two as e.g. W7-X where it was not considered to minimise the support structure mass. Another uncertainty is introduced by the fact that it is not everywhere clear whether the conductors and other structural elements within the winding packs (e.g. conductor jackets) are counted as structural material or not. It is clear from the figure, that with increasing stored magnetic energy the used support structure is getting closer to Viriral limit which represents the minimal required support structure from an energy point of view. This means that the support structure is optimised and used more efficiently with increasing stored energy. This can be understood as the support structure becomes a costing factor with increasing mass.

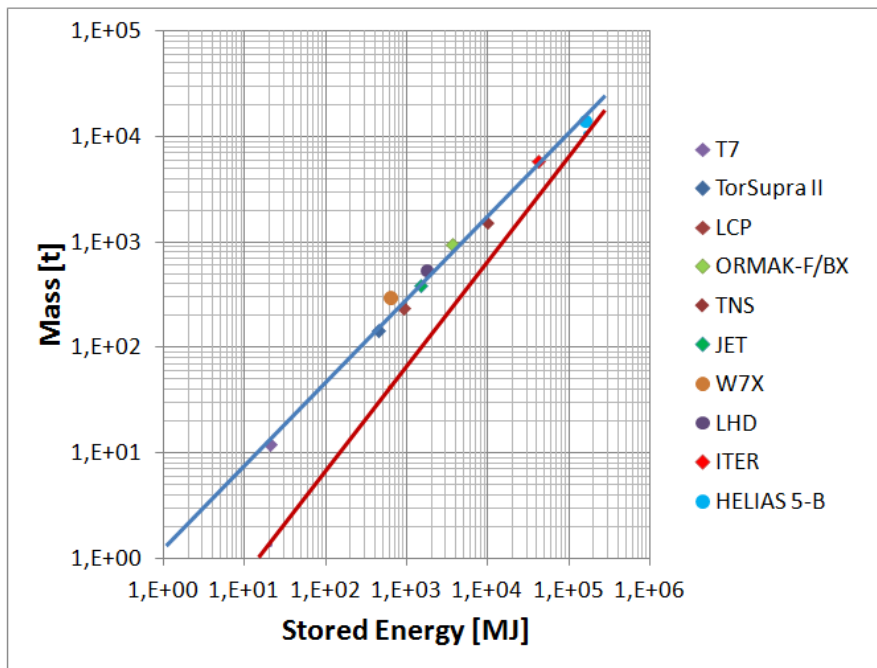

Figure 3: Virial limit of the required support structure with respect to magnetic energy (red line) and an empirical scaling (blue line) based on engineering designs of shown devices (colored rectangles).

Since the casing of the Helias 5-B coils is part of the support structure, and as the circumferential length of the coils is known from the calculations above, the crosssectional area of the coil casing can similarly be related to the magnetic energy. Is should be noted here, that based on the advanced and optimised support structure design of Helias 5-B it is intrinsically assumed in the coil model, that the high magnetic forces and stresses on the order of $650 \mathrm{MPa}$ are within allowable limits as investigated in [9, 17] in detail and not treated further here.

\subsection{Plasma Transport}

The current description of plasma transport in PROCESS is based on confinement time scalings. It is thereby possible to choose between different existing emprirical scalings which have been derived from experiments. Also for stellarators, several empirical confinement time scalings exist. The most recent is the so-called 'ISS04' scaling which has been obtained from combined experimentel 
data of the international stellarator heliotron confinement database [18. Such scalings can be easily integrated into the systems code PROCESS.

Alternatively to relying for the plasma transport description on empirical confinement time scalings derived 455 in parameter regimes which are outside the range of a reactor, the idea here is to follow a predictive ansatz based on available theoretical and numerical knowledge.

In this approach an uncertain, yet likely influential, part is the so-called anomalous transport which is dominant in stellarator experiments at the plasma edge 19. Since it is believed that the underlying mechanism to anomalous the plasma, complex 3D turbulence, gyrokinetic GENE simulations have been started for helical advanced stellarator geometries 20]. The goal thereby is the assessment of turbulent behaviour of e.g. ion-temperature-gradient intabilities (ITG) in 3D geometry. From such an analysis simplified 1D models may be developed compatible with well-established neoclassical transport simulations [21, 22]. With such a combination of neoclassical and turbulent transport, predictive physics scenarios can be simulated allowing one to derive the corresponding confinement times which may be compactly employed to describe the plasma transport in a systems code.

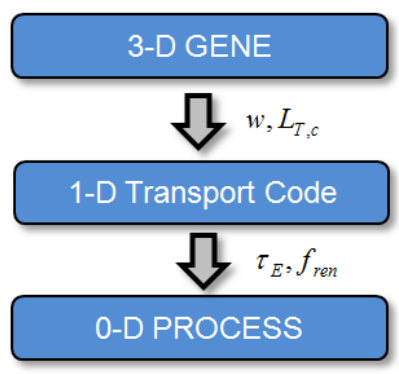

Figure 4: Startegy for predictive confinement time scaling development. The 3D GENE simulations for the ITG transport may allow one to derive a critical temperature gradient length $L_{T, c}$ and linear increase $w$ with respect to the gradient. These results can be combined with a $1 \mathrm{D}$ transport code to derive predictive confinement 470 times $\tau_{E}$ and a renormalisation factor $f_{r e n}$ with respect to empirical scalings.

This strategy is conceptually illustrated in Fig. 4. It was shown in 23. that the trapped electron modes (TEM) are $_{475}$ stabilised in helical advanced stellarator configurations in a large region of the parameter space. Instead, ITG modes may still contribute significantly to the plasma transport. For this reason the strategy for the predictive confinement time exploration concentrates on the ITG induced trans- ${ }_{480}$ port.

In tokamaks ITG transport is usually well described by a critical-gradient model. This means that the ITG modes are destabilised above a certain threshold of the temperature gradient length, $L_{T_{i}}^{-1}=-1 / T_{i} d T_{i} / d r$ (where ${ }_{485}$
$\omega_{T_{i}}=a / L_{T_{i}}$ (here, $a$ denotes the averaged minor radius of the stellarator). Then, the ion heat diffusivity defined as $\left\langle Q_{i}\right\rangle / \omega_{T_{i}}$ is found from the GENE simulations to increase almost linearly (at least well above marginality) with respect to the gradient $\omega_{T_{i}}$ (the brackets $\langle\cdots\rangle$ denote averaging with respect to the simulation box and time). The critical gradient $L_{T_{i}, c}$ and the slope of the linear fit are readily derived as illustrated by the example in Fig. 5.

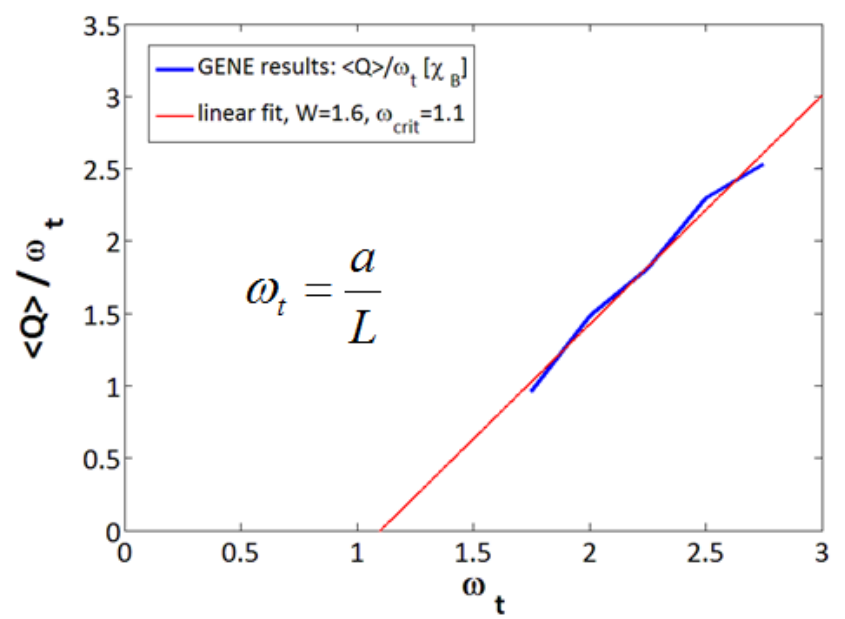

Figure 5: Scaling of the ion heat diffusivity as a function of the ion temperature gradient from GENE simulations (blue) and a linear fit (red).

It should be noted that the inclusion of a density gradi60 ent and the equilibrium radial electric field is lacking in the present results. Preliminary studies for W7-AS data, however, show a significant reduction of the ITG instability with increasing density gradient. The same is true for the radial electric field, as long as the sign of the electric field is favourable (in the opposite case, a further amplification of the ITG dynamics might occur; detailed calculations are ongoing).

Nonetheless, an exemplary application of the neoclassical 1D transport code with an ITG critical gradient model shall be demonstrated here. For this purpose two simulations are carried out. To put the results of the simulation with the ITG model in relation, the first simulation employs for comparison an empirical anomalous transport model which has been obtained from W7-AS where the diffusion coefficient scales with the absorbed power and inversely with the density, $\chi_{\mathrm{a}} \sim P^{3 / 4} n^{-1}[24,25]$. For this first simulation the diffusion coefficient has been adjusted to be on the order of $1 \mathrm{~m}^{2} / \mathrm{s}$ at the plasma edge. The second simulation employs the ITG critical gradient model where the diffusion coefficient scales with the ion temperature gradient using the corresponding values obtained from the GENE calculations. For both simulations presented here, as additional boundary condition, the temperature at the edge was chosen to be below $100 \mathrm{eV}$ since the strong temperature pedestals found in tokamak $\mathrm{H}$-mode discharges have yet to be observed in stellarators. 

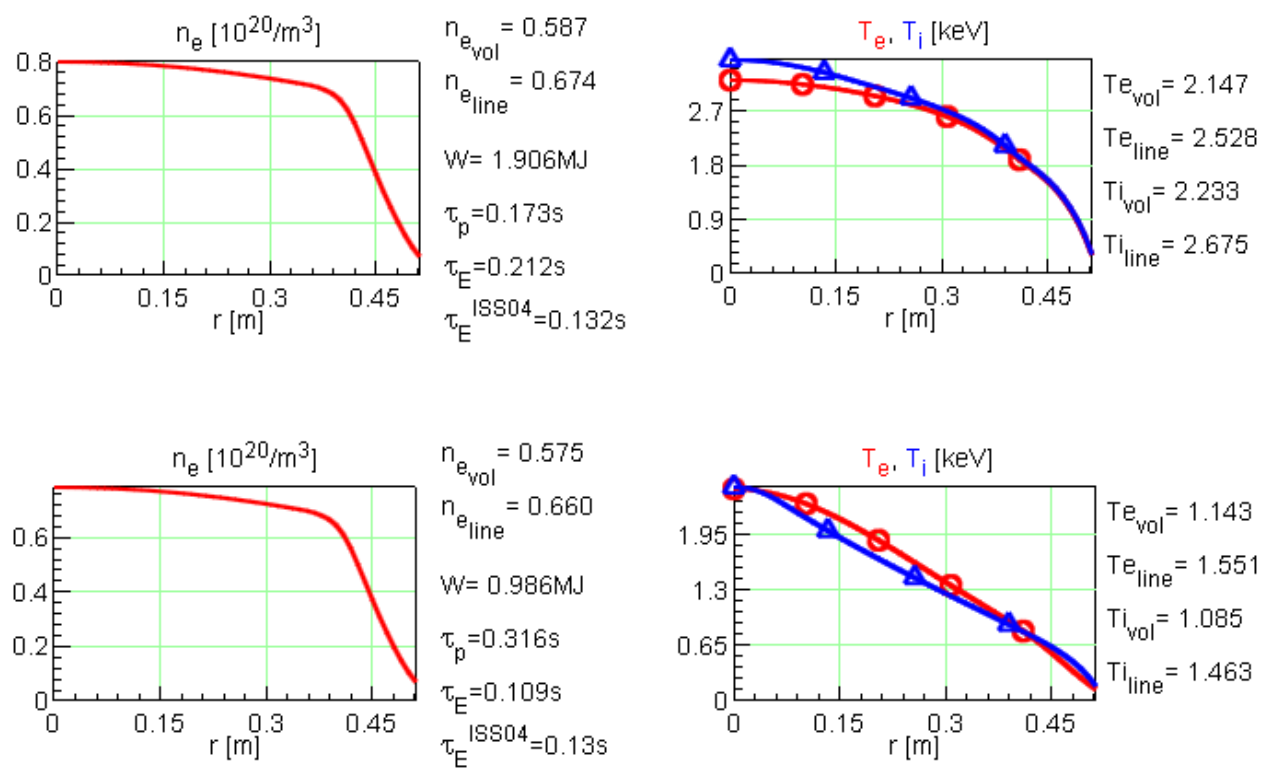

Figure 6: Transport model study: Comparison of a neoclassical 1D transport simulation employing an empirical anomalous transport model obtained from W7-AS (up) and an ITG critical gradient model (bottom). Both simulations are done for $P_{N B I}=10$ MW heating power and similarly flat density profiles (left). Next to the figures, also the corresponding line- and volume-averaged densities and temperatures can be found as well as particle and energy confinement times.

For both simulations, the most reactor-relevant, W7-X high-mirror configuration has been chosen and both simulations were carried out with the same fixed density profile (neglecting fuelling and particle exhaust issues) with a central electron density of $0.810^{20} \mathrm{~m}^{-3}$. For compatibility 505 also the heating scheme for both simulations was chosen to be $10 \mathrm{MW}$ neutral beam injection. This heating scheme was selected in order to heat the ions and reach significant ion temperature gradients. The resulting temperature profiles are shown in Fig. 6 and the corresponding neoclassical ${ }_{510}$ and anomalous diffusion coefficients are illustrated in Fig. 7.
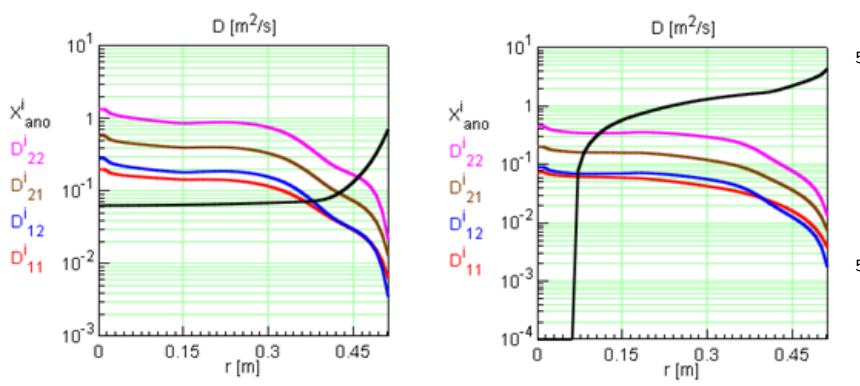

Figure 7: Comparison of neoclassical (coloured) and anomalous diffu- ${ }^{525}$ sion coefficients (black) for an empirical anomalous transport model obtained from W7-AS (left) and an ITG critical gradient model (right).

As the neoclassical effects were an optimisation crite-530 transport has been minimised to a level were it becomes comparable to turbulent transport. It can be seen from Fig. 7 that ITG turbulence may thus be the dominating transport channel over the whole plasma. This would lead to 'stiff' temperature profiles well-known from tokamaks as can be inferred from Fig. 6 (bottom-right). The ITG simulation here showed that the performance of the plasma in terms of confinement time and central temperature is a factor 2 below the neoclassical simulation which employs an empirical anomalous transport model.

It should be noted, that in tokamaks 'stiff' temperature profiles are usually observed in combination with a strong edge pedestal structure. Such large structures are not seen in stellaratros and are therefore excluded in the simulation here. But depending on the assumptions on a pedestal the anomalous diffusion profile would shift which in turn would have impact on plasma transport and performance.

Interestingly, to date 'stiff' temperature profiles have not been observed in stellarator experiments. This discrepancy with the presented results might admit several explanations, the most probable of which is the use of a local (flux-tube) model for the simulations, which is not able to capture the overall geometrical effects on the magnetic surface. Indeed, incorporating such an information (which is outside the scope of the present investigation) provides a milder heat-flux scaling to the one obtained here, in view of the dependence on the normalized ion gyroradius $\rho_{i} / a$, which is peculiar to stellarators only.

As already noted, also the inclusion of a density gradient and the equilibrium radial electric field is lacking in the present results. A significant reduction of the ITG in- 
stability is expected with increasing density gradient and the radial electric field, as long as the sign of the electric field is favourable. Further improvements in terms of the density gradient and the equilibrium electric field might590 also alleviate these differences and will be addressed in a future work.

\section{Summary}

HELIAS-specific models were developed for a systems code approach, especially with respect to modular systems codes as e.g. PROCESS. The main differences between ${ }^{595}$ the tokamak and the helical advanced stellarator concept have been reviewed. Since the stellarator is a steady-state device working without plasma current, the poloidal magnetic field must be created by the external coils. This implies a complex three-dimensional plasma shape as defined ${ }^{600}$ by flux surfaces as well as non-planar coils of several different geometries. The $3 \mathrm{D}$ shape of the plasma introduces additional localised particle orbits which cause significant neoclassical transport. The naturally occurring magnetic islands at the edge lead to a discontinuous island divertor concept.

By thorough comparison and review of these consider-605 ations with the models found in the well-established systems code PROCESS, four independent, specific models were identified for which stellarator-specific developments are required. By taking into account that systems codes $^{610}$ models should require low calculation times while preserving the stellarator complexity, up to now the following three models have been successfully developed. A geometry model based on Fourier coefficients which can repre- ${ }^{615}$ sent the complex 3D plasma shape, a basic island divertor model which assumes diffusive cross-field transport and high radiation at the $\mathrm{X}$-point and a coil model based on the Helias 5-B design in combination with inductance and ${ }^{620}$ field calculations.

The implementation of the proposed stellarator module to the systems code PROCESS and its verification is described in detail in a separate work [10. In the men- ${ }^{625}$ tioned work the HELIAS models are tested with respect to W7-X and, exploiting the generality of the models, with respect to a tokamak DEMO reference case. Both benchmarks exhibit very good agreement, justifying the use of ${ }^{630}$ the HELIAS module for future systems studies.

Beyond that, a transport description strategy has been developed which is anticipated to employ a confinement time scaling derived from sophisticated $1 \mathrm{D}$ neoclassical $^{635}$ and 3D turbulence simulations. Using gyrokinetic GENE simulations, critical parameters for the important iontemperature-gradient mode can be obtained. With these simulations it is possible to develop a critical gradient ${ }^{640}$ model compatible with the 1D transport code. Although a systematic study of the ITG transport in W7-X with respect to the density gradient length and the electric field is still under investigation, the basic principle of the strategy could be demonstrated by comparison of an empirical anomalous transport model with a 'worst-case' ITG transport model. The developed module will now be employed for detailed parameter studies for upscaled HELIAS configurations.

\section{Acknowledgments}

The authors thank D. Ward, P. Knight and R. Kemp of the Culham Centre for Fusion Energy for the introductions, explanations and fruitful discussions regarding the systems code PROCESS.

The GENE simulations have been performed at the Helios supercomputer (Japan).

This work has been carried out within the framework of the EUROfusion Consortium and has received funding from the European Union's Horizon 2020 research and innovation programme under grant agreement number 633053 . The views and opinions expressed herein do not necessarily reflect those of the European Commission.

\section{References}

[1] B. J. Green. "ITER: burning plasma physics experiment." Plasma Physics and Controlled Fusion, vol. 45, p. 687 (2003).

[2] F. Najmabadi and The ARIES Team. "The ARIES-AT advanced tokamak, Advanced technology fusion power plant." Fusion Engineering and Design, vol. 80, p. 3 (2006).

[3] D. Maisonnier, D. Campbell, I. Cook et al. "Power plant conceptual studies in Europe." Nuclear Fusion, vol. 47, p. 1524 (2007).

[4] D. J. Ward. "Newly Developing Conceptions of DEMOs: Pulsing and Hydrogen." Fusion Science and Technology, vol. 56, p. $581(2009)$.

[5] H. Zohm. "On the minimum size of DEMO." Fusion Science and Technology, vol. 58, p. 613 (2010).

[6] T. Goto, Y. Suzuki, N. Yanagi et al. "Importance of helical pitch parameter in LHD-type heliotron reactor designs." $\mathrm{Nu}$ clear Fusion, vol. 51, p. 083045 (2011).

[7] A. Sagara, T. Goto, J. Miyazawa et al. "Design activities on helical DEMO reactor FFHR-d1." Fusion Engineering and Design, vol. 87, p. 594 (2012).

[8] A. Sagara, H. Tamura, T. Tanaka et al. "Helical reactor design FFHR-d1 and c1 for steady-state DEMO." Fusion Engineering and Design, vol. 89, p. 2114 (2014).

[9] F. Schauer, K. Egorov and V. Bykov. "HELIAS 5-B magnet system structure and maintenance concept." Fusion Engineering and Design, vol. 88, p. 1619 (2013).

[10] F. Warmer, C. D. Beidler, A. Dinklage et al. "Implementation and Verification of a HELIAS module for the Systems Code PROCESS." Fusion Engineering and Design, vol. XX, p. YY (2014).

[11] Y. Feng. "Up-scaling the island divertor along the W7stellarator line." Journal of Nuclear Materials, vol. 438, p. S497 (2013).

[12] Y. Feng, M. Kobayashi, T. Lunt et al. "Comparison between stellarator and tokamak divertor transport." Plasma Physics and Controlled Fusion, vol. 53, p. 024009 (2011).

[13] S. Babic, F. Sirois, C. Akyel et al. "Mutual Inductance Calculation Between Circular Filaments Arbitrarily Positioned in Space: Alternative to Grovers Formula." Transaction on Magnetics, vol. 46, p. 3591 (2010).

[14] B. Montgomery. Solenoid Magnet Design. Wiley-Interscience, New York (1969).

[15] Y. Ilyin, A. Nijhuis and E. Krooshoop. "Scaling law for the strain dependence of the critical current in an advanced 
ITER $\mathrm{Nb}_{3}$ Sn strand." Superconductor Science and Technology, vol. 20, p. 186 (2007).

650 [16] F. C. Moon. "The virial theorem and scaling laws for superconducting magnet systems." Journal of Applied Physics, vol. 53, p. 9112 (1982).

[17] F. Schauer. "Coil winding pack FE-analysis for a HELIAS reactor." Fusion Engineering and Design, vol. 86, p. 636 (2011).

655 [18] H. Yamada, J. H. Harris, A. Dinklage et al. "Characterization of energy confinement in net-current free plasmas using the extended International Stellarator Database." Nuclear Fusion, vol. 45 , p. 1684 (2005).

[19] A. Dinklage, M. Yokoyama, K. Tanaka et al. "Inter-machine validation study of neoclassical transport modelling in mediumto high-density stellarator-heliotron plasmas." Nuclear Fusion, vol. 53, p. 063022 (2013).

[20] P. Xanthopoulos, F. Merz, T. Grler et al. "Nonlinear Gyrokinetic Simulations of Ion-Temperature-Gradient Turbulence for the Optimized Wendelstein 7-X Stellarator." Physical Review Letters, vol. 99, p. 035002 (2007).

[21] C. D. Beidler, K. Allmaier, M. Y. Isaev et al. "Benchmarking of the mono-energetic transport coefficients - results from the International Collaboration on Neoclassical Transport in Stellarators (ICNTS)." Nuclear Fusion, vol. 51, p. 076001 (2011).

[22] Y. Turkin, C. D. Beidler, H. Maaßberg et al. "Neoclassical transport simulations for stellarators." Physics of Plasmas, vol. 18, p. 022505 (2011).

[23] J. H. E. Proll, P. Helander, J. W. Connor et al. "Resilience of Quasi-Isodynamic Stellarators against Trapped-Particle Instabilities." Physical Review Letters, vol. 108, p. 245002 (2012).

[24] H. Maaßberg, R. Brakel, R. Burhenn et al. "Transport in stellarators." Plasma Physics and Controlled Fusion, vol. 35, p. B319 (1993).

${ }_{680}[25]$ U. Stroth. "A comparative study of transport in stellarators and tokamaks." Plasma Physics and Controlled Fusion, vol. 40, p. 9 (1998). 